\title{
Maggot Debridement Therapy (MDT) for Treatment of Cutaneous Leishmaniasis Wound using Lucilia serricata Larvae in Iran: Case Reports
}

\author{
Asghar Nasiri 1(D), Elham Jahanifard ${ }^{2}(\mathbb{D})$, Mona Sharififard ${ }^{2 *}(D)$, Reza Arjmand ${ }^{3(D)}$, \\ Sima Rasai 4 (iD), Tahmaseb Haeri ${ }^{5}$ (iD
}

1. Student Research Committee, Ahvaz Jundishapur University of Medical Sciences, Ahvaz, Iran

2. Dept. of Medical Entomology and Vector Control, School of Public Health, Ahvaz Jundishapur University of Medical Sciences, Ahvaz, Iran

3. Dept. of Medical Parasitology, School of Medicine, Ahvaz Jundishapur University of Medical Sciences, Ahvaz, Iran

4. Dept. of Dermatology, School of Medicine, Ahvaz, Jundishapur University of Medical Sciences, Ahvaz, Iran

5. Health Center of Andimeshk County, Ahvaz Jundishapur University of Medical Sciences, Ahvaz, Iran

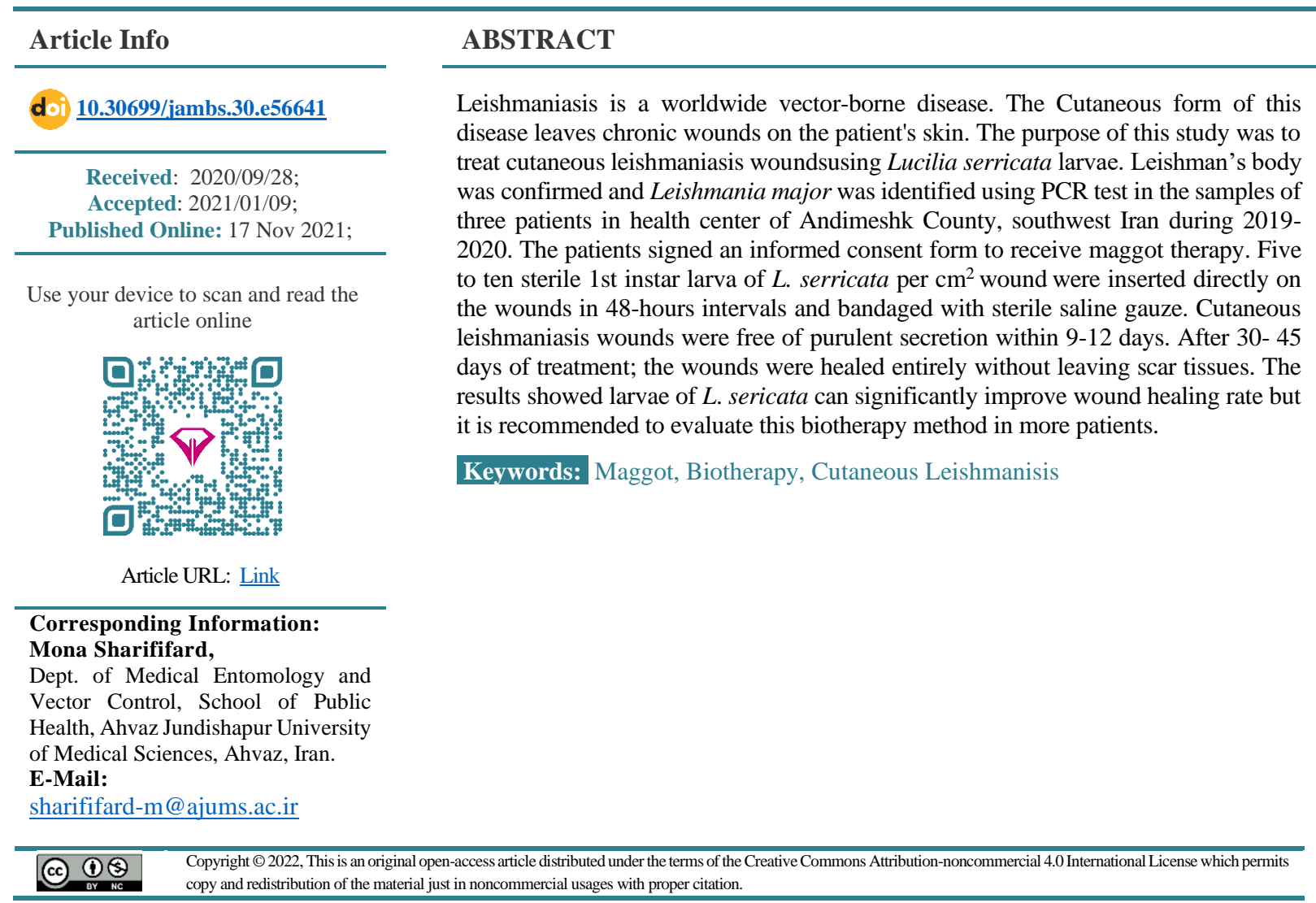

\section{Introduction}

Leishmaniasis as a vector-borne disease is one of the worldwide zoonotic diseases caused by a variety of intracellular protozoan parasites that target mammalian macrophages and are transmitted by different species of Phlebotomus and Lutzomyia sand flies (1-2). The disease has three clinical forms including cutaneous, mucocutaneous and visceral forms. It was reported from 98 countries on four continents. Coetaneous leishmaniasis (CL) and visceral leishmaniasis (VL), the two common forms of the disease, are endemic in 92 and 83 countries according to a WHO report in 2018. The prevalence and the incidence rates of CL are 12 million and 1-1.5 million respectively and indices rate of VL is 0.5 million (1).
Cutaneous leishmaniasis is commonly seen in Iran, and Khuzestan Province is an endemic area for zoonotic cutaneous leishmaniasis (ZCL) (3). Leishmania tropica and $L$. major are etiological agents of $C L$, and infection causes skin sores. At first, papules or nodules usually appear on the body especially on the face, hands and feet that are exposed to female sandfly bites. The sores may end up as infectious ulcers and they leave permanent scars after healing (4-6). The first-line drugs for treating cutaneous leishmaniasis are pentavalent antimonials [sodium stibogluconate (pentostam) or meglumine antimoniate (glucantime)]. Currently, for some reasons, such as inadequate effectiveness of the common drugs, the lack of patient accompanying in completing the treatment period, and the problems of providing drugs for 
the governments, finding more effective, cost-effective and safer treatment alternative is considered as a serious necessity $(4,7)$.

The Use of sterile fly maggots for the treatment of chronic wounds is named maggot debridement therapy (MDT). The most common species has been used in MDT so far is Lucilia serricata (Calliphoridae). These fly larvae secrete proteolytic enzymes, antibacterial secretions, and some substances that stimulate granulation in necrotic tissues when they pass from the 2 nd stage to the $3^{\text {rd }}$ stage. These secretions lyse, kill or inhibit the bacteria, thus disinfecting the lesions. As a standard, applying sterile fly larvae at a density of five to eight per $\mathrm{cm}^{2}$ to the wound is recommended (8).

Recent studies have shown the effectiveness of maggot therapy in wound healing on animal and human hosts $(2,4-10)$. The purpose of this study was to treat human cutaneous leishmaniasis wound using $L$. serricata maggot.

\section{Ethical issues:}

This project confirmed in the Ethical Committee of Ahvaz Jundishapur University of Medical Sciences and received cod number IRAJUMS.REC.1398.767. Furthermore, informed consents designed by the university were signed by all patients that took part in the project. In the all cases, leishman body was confirmed in Pathobiology sample and determined as L. major using PCR.

\section{Case Reports}

Case 1: A 52-year-old man with a $2.5 \mathrm{~cm}$ diameter infectious wound on his right forearm was referred to the health center of Andimeshk County, southwest Iran in June 2019. He was a rancher. Leishmania body was confirmed in his Pathobiology sample and determined as Leishmania major using the PCR test. The patient was informed about the treatments and signed an informed consent form to receive maggot therapy. Ten sterile $1^{\text {st }}$ instar larva of Lucilia serricata were inserted directly on the wound and then bandaged with saline moistened gauze based on MDT protocol (8) in two 48-hour cycles. After the second larval application, the wound was free of purulent secretion, and symptoms of healing appeared. Fly maggot had grown from $2-3 \mathrm{~mm}$ to $10-12 \mathrm{~mm}$ in size during the treatment. The patient's wound was treated within nine days. The location of the lesion was completely healed without leaving scar tissues 45 days after treatment (Figure 1).

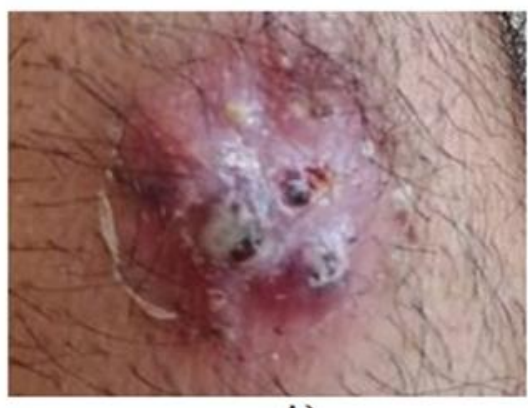

A)

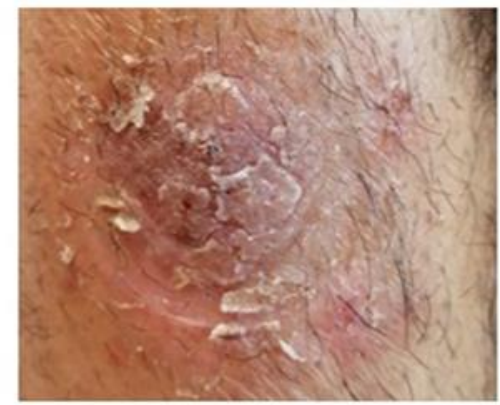

D)

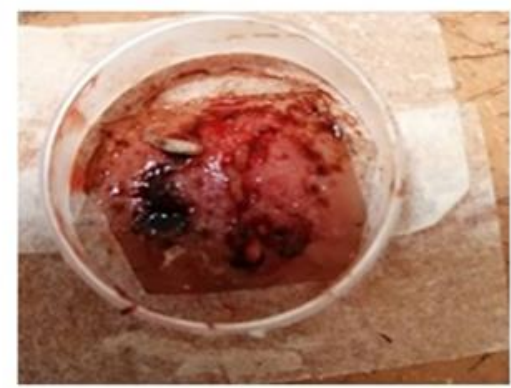

B)

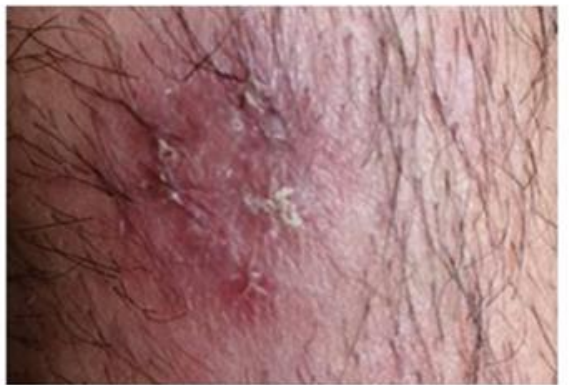

E)

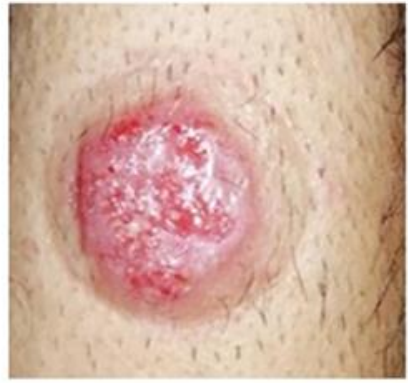

C)

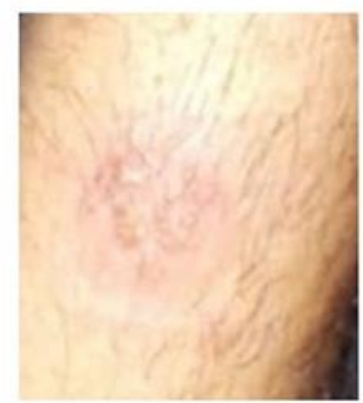

F)

Figure 1: Before maggot therapy, B) During maggot therapy, C) Immediately after removing the second maggot dressing, D) Two weeks later, E) After one month, D) After 45 days

Case 2: A 23- years-old solder with a $2 \mathrm{~cm}$ diameter infectious wound on his left forearm was referred to the health center of Andimeshk County in September 2019. Leishmania body was confirmed in Pathobiology sample and determined as L. major using PCR. The patient signed an informed consent form to receive maggot therapy. Ten sterile $1^{\text {st }}$ instar larva of $L$. serricata were placed directly on the wound in three 48-hour intervals. The wound was bandaged with saline sterile gauze after each larval insertion. After $48 \mathrm{~h}$, the wound bandage was removed, and the maggots were then extracted from the wound with sterile forceps. During 
the maggot therapy, the patient suffered from mild pain, which was prescribed by a mild painkiller by the physician. After the third larval application, the wound was free of purulent secretion, and symptoms of healing appeared. The patient's wound was treated in 12 days. He was followed up for 30 days to complete the healing of the wound without leaving scar tissues.

Case 3: A 16-years old girl with five wounds on her right forearm was referred to the health center of Andimeshk County in August 2019. She was a student and lived in the city. The maximum diameter of her wounds was $0.5 \mathrm{~cm}$, and the wounds were close together. Leishmania body was confirmed in the Pathobiology sample and determined as L. major using the PCR test. The patient signed an informed consent form to receive maggot therapy. Approximately 10-15 maggots were carefully inserted in the center wound with sterile forceps and gauze moistened with saline was applied on top of the maggots. Ten sterile $1^{\text {st }}$ instar larva of $L$. serricata were placed directly on the wound in three 48-hour intervals. In this case, the larvae were placed on open wounds, but all five wounds were bandaged with sterile saline gauze. Interestingly, the larva attacked all the wounds and evacuated them from purulent secretion. During the treatment, the patient suffered from mild pain, which was prescribed by a mild painkiller by the physician. The wounds were treated after nine days and completely healed without leaving scar tissues in at 45 days.

\section{Discussion}

Insects and their derived products including fly maggot, honey bee, and its derivatives (honey, royal jelly, propolis, venom), cantharidin, and ant's venom have been widely used in human healing worldwide since ancient times (11). The use of fly larvae to debride necrotic tissue undergoing maggot therapy or biosurgery is going back to human civilization. The maggots have been used to heal chronic and post-surgical wounds such as diabetic wounds, pressure lesions, superficial infectious tumors, and any infectious wounds or lesions (12). The mean duration treatment of CL with Glucantime was assessed $6.8 \pm 1.7$ weeks (4). The results of this study were remarkable, and the CL wound healing in MT was faster than routine therapies. The infectious wound was wholly treated by applying two series of 10 first instar larvae of L. serricata during 9-12 days, and it left no scar tissue in two months after treatment.

Polat et al. (2016) treated 29 patients with Glucantimeresistant/tolerant cutaneous leishmaniasis wounds with $L$. sericata larvae and its larval secretions efficiently without leaving any scare tissues. Additionally, after 1-2 months of treatment, Leishmania spp. was not detected in their patient's samples using PCR. This study confirms our results (4).

Other studies on the use of maggot therapy in the treatment of cutaneous leishmaniasis wounds have been limited to laboratory studies on animal hosts (2,5-7, 9-10). All of which confirms the notable therapeutic effect of this biosurgery method in the treatment of cutaneous leishmaniasis ulcers.

The efficacy of this method in the treatment of widely infectious wounds such as pressure wounds, diabetes, and contagious tumors has been proven previously $(8,15-16)$. Mirabzadeh et al. (2017) treated diabetic foot ulcers using maggot therapy in 28 patients. They reported MT as an advanced and accepted wound healing method (16).

\section{Conclusion}

Maggot debridement therapy is a part of the larger branch of medicine called biomedicine, in which living organisms such as leeches, bees, or fly larva are used directly for treatment. Our result showed that in all 3 cases, the wounds healed without leaving scar tissue at 3045 days but it is recommended to evaluate this biotherapy method in more patients.

\section{Acknowledgment}

This article was financially supported by Ahvaz Jundishapur University of Medical Sciences with proposal number of $98 \mathrm{~S} 72$ and ethical code of IRAJUMS.REC.1398.767 .

\section{Conflicts of Interest}

There is no conflict of interest.

\section{References}

1. Alvar J, Velez ID, Bern C, Herrero M, Desjeux P, Cano J, Jannin J, den Boer M, WHO Leishmaniasis Control Team. Leishmaniasis: worldwide and global estimates of its incidence. PloS one. 2012; 7(5): e35671. [DOI:10.1371/journal.pone.0035671] [PMID] [PMCID]

2. Sanei-Dehkordi A, Khamesipour A, Akbarzadeh K, Akhavan AA, Mohammadi AM, Mohammadi Y, Rassi Y, Oshaghi MA, Alebrahim Z, Eskandari SE, Rafinejad J. Anti Leishmania activity of Lucilia sericata and Calliphora vicina maggots in laboratory models. Exp. Parasitol. 2016; 170: 5965. [DOI:10.1016/j.exppara.2016.08.007] [PMID]

3. Jahanifard E, Hanafi-Bojd AA, Nasiri H, Matinfar HR, Charrahy Z, Abai MR, Yaghoobi-Ershadi MR, Akhavan AA. Prone Regions of Zoonotic Cutaneous Leishmaniasis in Southwest of Iran: Combination of Hierarchical Decision Model (AHP) and GIS. J Arthropod Borne Dis. 2019; 13(3):310. [DOI:10.18502/jad.v13i3.1540] [PMID] [PMCID]

4. Polat E, Kutlubay Z, Sirekbasan S. Treatment of Glucantime-resistant/tolerant cutaneous leishmaniasis with Lucilia sericata larvae and its 
larval secretions: The first study in the world. Trop. Biomed. 2016; 33(4): 668-74.

5. Cruz-Saavedra L, Díaz-Roa A, Gaona MA, Cruz ML, Ayala M, Cortés-Vecino JA, Patarroyo MA, Bello FJ. The effect of Lucilia sericata and Sarconesiopsis magellanica derived larval therapy on Leishmania panamensis. Acta Trop. 2016; 164: 280-9. [DOI:10.1016/j.actatropica.2016.09.020] [PMID]

6. Kabiri M, Dayer MS, Ghaffarifar F. Therapeutic Effects of Lucilia sericata Larvae on Cutaneous Leishmaniasis Wounds Caused by Leishmania major using BALB/c Mice as Animal Model. JKUMS. 2017; 24(5): 389-96.

7. Laverde-Paz MJ, Echeverry MC, Patarroyo MA, Bello FJ. Evaluating the anti-leishmania activity of Lucilia sericata and Sarconesiopsis magellanica blowfly larval excretions/secretions in an in vitro model. Acta Trop. 2018; 177: 44-50. [DOI:10.1016/j.actatropica.2017.09.033] [PMID]

8. Sherman RA. Maggot versus conservative debridement therapy for the treatment of pressure ulcers. Wound Repair Regen. 2002; 10(4): 208-14. [DOI:10.1046/j.1524-475X.2002.10403.x] [PMID]

9. Arrivillaga J, Rodríguez J, Oviedo M. Preliminary evaluation of maggot (Diptera: Calliphoridae) therapy as a potential treatment for leishmaniasis ulcers. Biomedica. 2008; 28(2):305-10. [DOI:10.7705/biomedica.v28i2.102] [PMID]

10. Polat E, Cakan H, Aslan M, Sirekbasan S, Kutlubay Z, Ipek T, Ozbilgin A. Detection of antileishmanial effect of the Lucilia sericata larval secretions in vitro and in vivo on Leishmania tropica: first work. Exp Parasitol. 2012; 132(2): 129-34. [DOI:10.1016/j.exppara.2012.06.004] [PMID]

11. Cherniack EP. Bugs as Drugs, Part 1: Insects: the" new" alternative medicine for the 21st century. Alter Med Rev. 2010; 15(2):124-35.

12. Whitaker IS, Twine C, Whitaker MJ, Welck M, Brown CS, Shandall A. Larval therapy from antiquity to the present day: mechanisms of action, clinical applications and future potential. Postgrad Med J. 2007; 83 (980): 409-13. http://dx.doi.org/10.1136/pgmj.2006.055905 [DOI:10.1136/pgmj.2006.055905] [PMCID]

13. Jaffary F, Nilforoushzadeh MA, Siadat A, Haftbaradaran E, Ansari N, Ahmadi E. A comparison between the effects of glucantime, topical trichloroacetic acid $50 \%$ plus glucantime, and fractional carbon dioxide laser plus glucantime on cutaneous leishmaniasis lesions. Dermatol Res Pract. http://dx.doi.org/10.1155/2016/6462804 [DOI:10.1155/2016/6462804] [PMID] [PMCID]

14. Paul AG, Ahmad NW, Lee HL, Ariff AM, Saranum M, Naicker AS, Osman Z. Maggot debridement therapy with Lucilia cuprina: a comparison with conventional debridement in diabetic foot ulcers. Int Wound J. 2009; 6(1):3946. [DOI:10.1111/j.1742-481X.2008.00564.x] [PMID] [PMCID]

15. Gericke A, Hoffmann EM, Pitz S, Pfeiffer N. Maggot therapy following orbital exenteration. $\mathrm{Br}$ J Ophthalmol. 2007; 91(12):1715-6. [DOI:10.1136/bjo.2007.116673] [PMID] [PMCID]

\section{How to Cite This Article:}

Nasiri A, Jahanifard E, Sharififard M, Arjmand R, Rasai S, Haeri T. Maggot Debridement Therapy (MDT) for Treatment of Cutaneous Leishmaniasis Wound using Lucilia serricata Larvae in Iran: Case Reports. J Adv Med Biomed Res. 2022; 30 (138) :69-72

\section{Download citation:}

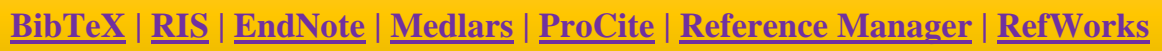

